\title{
The Lebesgue Monotone Convergence Theorem
}

\author{
Noboru Endou \\ Keiko Narita \\ Gifu National College of Technology \\ Hirosaki-city \\ Japan \\ Aomori, Japan \\ Yasunari Shidama \\ Shinshu University \\ Nagano, Japan
}

\begin{abstract}
Summary. In this article we prove the Monotone Convergence Theorem $[16]$.
\end{abstract}

MML identifier: MESFUNC9, version: $\underline{7.8 .10 \quad 4.100 .1011}$

The notation and terminology used in this paper have been introduced in the following articles: [10], [20], [2], [7], [21], [6], [8], [9], [1], [17], [18], [3], [4], [5], [13], [14], [15], [19], [11], [12], and [22].

\section{Preliminaries}

For simplicity, we adopt the following rules: $X$ is a non empty set, $S$ is a $\sigma$-field of subsets of $X, M$ is a $\sigma$-measure on $S, E$ is an element of $S, F, G$ are sequences of partial functions from $X$ into $\overline{\mathbb{R}}, I$ is a sequence of extended reals, $f, g$ are partial functions from $X$ to $\overline{\mathbb{R}}, s_{1}, s_{2}, s_{3}$ are sequences of extended reals, $p$ is an extended real number, $n, m$ are natural numbers, $x$ is an element of $X$, and $z, D$ are sets.

Next we state a number of propositions:

(1) If $f$ is without $+\infty$ and $g$ is without $+\infty$, then $\operatorname{dom}(f+g)=\operatorname{dom} f \cap$ $\operatorname{dom} g$.

(2) If $f$ is without $+\infty$ and $g$ is without $-\infty$, then $\operatorname{dom}(f-g)=\operatorname{dom} f \cap$ dom $g$. 
(3) If $f$ is without $-\infty$ and $g$ is without $-\infty$, then $f+g$ is without $-\infty$.

(4) If $f$ is without $+\infty$ and $g$ is without $+\infty$, then $f+g$ is without $+\infty$.

(5) If $f$ is without $-\infty$ and $g$ is without $+\infty$, then $f-g$ is without $-\infty$.

(6) If $f$ is without $+\infty$ and $g$ is without $-\infty$, then $f-g$ is without $+\infty$.

(7)(i) If $s_{1}$ is convergent to finite number, then there exists a real number $g$ such that $\lim s_{1}=g$ and for every real number $p$ such that $0<p$ there exists a natural number $n$ such that for every natural number $m$ such that $n \leq m$ holds $\left|s_{1}(m)-\lim s_{1}\right|<p$,

(ii) if $s_{1}$ is convergent to $+\infty$, then $\lim s_{1}=+\infty$, and

(iii) if $s_{1}$ is convergent to $-\infty$, then $\lim s_{1}=-\infty$.

(8) If $s_{1}$ is non-negative, then $s_{1}$ is not convergent to $-\infty$.

(9) If $s_{1}$ is convergent and for every natural number $k$ holds $s_{1}(k) \leq p$, then $\lim s_{1} \leq p$.

(10) If $s_{1}$ is convergent and for every natural number $k$ holds $p \leq s_{1}(k)$, then $p \leq \lim s_{1}$.

(11) Suppose that

(i) $s_{2}$ is convergent,

(ii) $s_{3}$ is convergent,

(iii) $s_{2}$ is non-negative,

(iv) $s_{3}$ is non-negative, and

(v) for every natural number $k$ holds $s_{1}(k)=s_{2}(k)+s_{3}(k)$.

Then $s_{1}$ is non-negative and convergent and $\lim s_{1}=\lim s_{2}+\lim s_{3}$.

(12) Suppose for every natural number $n$ holds $G(n)=F(n)\lceil D$ and $x \in D$. Then

(i) if $F \# x$ is convergent to $+\infty$, then $G \# x$ is convergent to $+\infty$,

(ii) if $F \# x$ is convergent to $-\infty$, then $G \# x$ is convergent to $-\infty$,

(iii) if $F \# x$ is convergent to finite number, then $G \# x$ is convergent to finite number, and

(iv) if $F \# x$ is convergent, then $G \# x$ is convergent.

(13) If $E=\operatorname{dom} f$ and $f$ is measurable on $E$ and $f$ is non-negative and $M(E \cap \mathrm{EQ}-\operatorname{dom}(f,+\infty)) \neq 0$, then $\int f \mathrm{~d} M=+\infty$.

(14) $\int \chi_{E, X} \mathrm{~d} M=M(E)$ and $\int \chi_{E, X}\lceil E \mathrm{~d} M=M(E)$.

(15) Suppose that

(i) $E \subseteq \operatorname{dom} f$,

(ii) $E \subseteq \operatorname{dom} g$,

(iii) $f$ is measurable on $E$,

(iv) $g$ is measurable on $E$,

(v) $f$ is non-negative, and

(vi) for every element $x$ of $X$ such that $x \in E$ holds $f(x) \leq g(x)$.

Then $\int f\left\lceil E \mathrm{~d} M \leq \int g\lceil E \mathrm{~d} M\right.$. 


\section{Selected Properties of Extended Real Sequence}

Let $f$ be an extended real-valued function and let $x$ be a set. Then $f(x)$ is an element of $\overline{\mathbb{R}}$.

Let $s$ be an extended real-valued function. The functor $\left(\sum_{\alpha=0}^{\kappa} s(\alpha)\right)_{\kappa \in \mathbb{N}}$ yields a sequence of extended reals and is defined by:

(Def. 1) $\left(\sum_{\alpha=0}^{\kappa} s(\alpha)\right)_{\kappa \in \mathbb{N}}(0)=s(0)$ and for every natural number $n$ holds $\left(\sum_{\alpha=0}^{\kappa} s(\alpha)\right)_{\kappa \in \mathbb{N}}(n+1)=\left(\sum_{\alpha=0}^{\kappa} s(\alpha)\right)_{\kappa \in \mathbb{N}}(n)+s(n+1)$.

Let $s$ be an extended real-valued function. We say that $s$ is summable if and only if:

(Def. 2) $\quad\left(\sum_{\alpha=0}^{\kappa} s(\alpha)\right)_{\kappa \in \mathbb{N}}$ is convergent.

Let $s$ be an extended real-valued function. The functor $\sum s$ yielding an extended real number is defined as follows:

(Def. 3) $\sum s=\lim \left(\left(\sum_{\alpha=0}^{\kappa} s(\alpha)\right)_{\kappa \in \mathbb{N}}\right)$.

Next we state several propositions:

(16) If $s_{1}$ is non-negative, then $\left(\sum_{\alpha=0}^{\kappa}\left(s_{1}\right)(\alpha)\right)_{\kappa \in \mathbb{N}}$ is non-negative and $\left(\sum_{\alpha=0}^{\kappa}\left(s_{1}\right)(\alpha)\right)_{\kappa \in \mathbb{N}}$ is non-decreasing.

(17) If for every natural number $n$ holds $0<s_{1}(n)$, then for every natural number $m$ holds $0<\left(\sum_{\alpha=0}^{\kappa}\left(s_{1}\right)(\alpha)\right)_{\kappa \in \mathbb{N}}(m)$.

(18) If $F$ has the same dom and for every natural number $n$ holds $G(n)=$ $F(n)\lceil D$, then $G$ has the same dom.

(19) Suppose that

(i) $D \subseteq \operatorname{dom} F(0)$,

(ii) for every natural number $n$ holds $G(n)=F(n)\lceil D$, and

(iii) for every element $x$ of $X$ such that $x \in D$ holds $F \# x$ is convergent.

Then $\lim F\lceil D=\lim G$.

(20) Suppose $F$ has the same dom and $E \subseteq \operatorname{dom} F(0)$ and for every natural number $m$ holds $F(m)$ is measurable on $E$ and $G(m)=F(m)\lceil E$. Then $G(n)$ is measurable on $E$.

(21) Suppose that

(i) $E \subseteq \operatorname{dom} F(0)$,

(ii) $G$ has the same dom,

(iii) for every element $x$ of $X$ such that $x \in E$ holds $F \# x$ is summable, and

(iv) for every natural number $n$ holds $G(n)=F(n)\lceil E$.

Let $x$ be an element of $X$. If $x \in E$, then $G \# x$ is summable. 


\section{Partial Sums of Functional Sequence and their Properties}

Let $X$ be a non empty set and let $F$ be a sequence of partial functions from $X$ into $\overline{\mathbb{R}}$. The functor $\left(\sum_{\alpha=0}^{\kappa} F(\alpha)\right)_{\kappa \in \mathbb{N}}$ yields a sequence of partial functions from $X$ into $\overline{\mathbb{R}}$ and is defined as follows:

(Def. 4) $\left(\sum_{\alpha=0}^{\kappa} F(\alpha)\right)_{\kappa \in \mathbb{N}}(0)=F(0)$ and for every natural number $n$ holds $\left(\sum_{\alpha=0}^{\kappa} F(\alpha)\right)_{\kappa \in \mathbb{N}}(n+1)=\left(\sum_{\alpha=0}^{\kappa} F(\alpha)\right)_{\kappa \in \mathbb{N}}(n)+F(n+1)$.

Let $X$ be a set and let $F$ be a sequence of partial functions from $X$ into $\overline{\mathbb{R}}$. We say that $F$ is additive if and only if:

(Def. 5) For all natural numbers $n, m$ such that $n \neq m$ and for every set $x$ such that $x \in \operatorname{dom} F(n) \cap \operatorname{dom} F(m)$ holds $F(n)(x) \neq+\infty$ or $F(m)(x) \neq-\infty$.

Next we state a number of propositions:

(22) If $z \in \operatorname{dom}\left(\sum_{\alpha=0}^{\kappa} F(\alpha)\right)_{\kappa \in \mathbb{N}}(n)$ and $m \leq n$, then $z \in$ $\operatorname{dom}\left(\sum_{\alpha=0}^{\kappa} F(\alpha)\right)_{\kappa \in \mathbb{N}}(m)$ and $z \in \operatorname{dom} F(m)$.

(23) If $z \in \operatorname{dom}\left(\sum_{\alpha=0}^{\kappa} F(\alpha)\right)_{\kappa \in \mathbb{N}}(n)$ and $\left(\sum_{\alpha=0}^{\kappa} F(\alpha)\right)_{\kappa \in \mathbb{N}}(n)(z)=+\infty$, then there exists a natural number $m$ such that $m \leq n$ and $F(m)(z)=+\infty$.

(24) If $F$ is additive and $z \in \operatorname{dom}\left(\sum_{\alpha=0}^{\kappa} F(\alpha)\right)_{\kappa \in \mathbb{N}}(n)$ and $\left(\sum_{\alpha=0}^{\kappa} F(\alpha)\right)_{\kappa \in \mathbb{N}}(n)(z)=+\infty$ and $m \leq n$, then $F(m)(z) \neq-\infty$.

(25) If $z \in \operatorname{dom}\left(\sum_{\alpha=0}^{\kappa} F(\alpha)\right)_{\kappa \in \mathbb{N}}(n)$ and $\left(\sum_{\alpha=0}^{\kappa} F(\alpha)\right)_{\kappa \in \mathbb{N}}(n)(z)=-\infty$, then there exists a natural number $m$ such that $m \leq n$ and $F(m)(z)=-\infty$.

(26) If $F$ is additive and $z \in \operatorname{dom}\left(\sum_{\alpha=0}^{\kappa} F(\alpha)\right)_{\kappa \in \mathbb{N}}(n)$ and $\left(\sum_{\alpha=0}^{\kappa} F(\alpha)\right)_{\kappa \in \mathbb{N}}(n)(z)=-\infty$ and $m \leq n$, then $F(m)(z) \neq+\infty$.

(27) If $F$ is additive, then $\left(\sum_{\alpha=0}^{\kappa} F(\alpha)\right)_{\kappa \in \mathbb{N}}(n)^{-1}(\{-\infty\}) \cap F(n+$ $1)^{-1}(\{+\infty\})=\emptyset$ and $\left(\sum_{\alpha=0}^{\kappa} F(\alpha)\right)_{\kappa \in \mathbb{N}}(n)^{-1}(\{+\infty\}) \cap F(n+1)^{-1}(\{-\infty\})=$ $\emptyset$.

(28) If $F$ is additive, then $\operatorname{dom}\left(\sum_{\alpha=0}^{\kappa} F(\alpha)\right)_{\kappa \in \mathbb{N}}(n)=\bigcap\{\operatorname{dom} F(k) ; k$ ranges over elements of $\mathbb{N}: k \leq n\}$.

(29) If $F$ is additive and has the same dom, then $\operatorname{dom}\left(\sum_{\alpha=0}^{\kappa} F(\alpha)\right)_{\kappa \in \mathbb{N}}(n)=$ $\operatorname{dom} F(0)$.

(30) If for every natural number $n$ holds $F(n)$ is non-negative, then $F$ is additive.

(31) If $F$ is additive and for every $n$ holds $G(n)=F(n)\lceil D$, then $G$ is additive.

(32) If $F$ is additive and has the same dom and $D \subseteq \operatorname{dom} F(0)$ and $x \in D$,

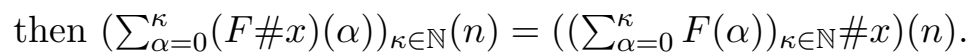

(33) Suppose $F$ is additive and has the same dom and $D \subseteq \operatorname{dom} F(0)$ and $x \in D$. Then

(i) $\quad\left(\sum_{\alpha=0}^{\kappa}(F \# x)(\alpha)\right)_{\kappa \in \mathbb{N}}$ is convergent to finite number iff $\left(\sum_{\alpha=0}^{\kappa} F(\alpha)\right)_{\kappa \in \mathbb{N}} \# x$ is convergent to finite number,

(ii) $\quad\left(\sum_{\alpha=0}^{\kappa}(F \# x)(\alpha)\right)_{\kappa \in \mathbb{N}}$ is convergent to $+\infty$ iff $\left(\sum_{\alpha=0}^{\kappa} F(\alpha)\right)_{\kappa \in \mathbb{N}} \# x$ is convergent to $+\infty$, 
(iii) $\quad\left(\sum_{\alpha=0}^{\kappa}(F \# x)(\alpha)\right)_{\kappa \in \mathbb{N}}$ is convergent to $-\infty$ iff $\left(\sum_{\alpha=0}^{\kappa} F(\alpha)\right)_{\kappa \in \mathbb{N}} \# x$ is convergent to $-\infty$, and

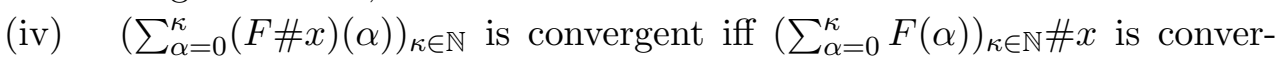
gent.

(34) If $F$ is additive and has the same $\operatorname{dom}$ and $\operatorname{dom} f \subseteq \operatorname{dom} F(0)$ and $x \in \operatorname{dom} f$ and $F \# x$ is summable and $f(x)=\sum F \# x$, then $f(x)=$ $\lim \left(\left(\sum_{\alpha=0}^{\kappa} F(\alpha)\right)_{\kappa \in \mathbb{N}} \# x\right)$.

(35) Suppose that for every natural number $m$ holds $F(m)$ is simple function in $S$. Then $F$ is additive and $\left(\sum_{\alpha=0}^{\kappa} F(\alpha)\right)_{\kappa \in \mathbb{N}}(n)$ is simple function in $S$.

(36) If for every natural number $m$ holds $F(m)$ is non-negative, then $\left(\sum_{\alpha=0}^{\kappa} F(\alpha)\right)_{\kappa \in \mathbb{N}}(n)$ is non-negative.

(37) If $F$ has the same dom and $x \in \operatorname{dom} F(0)$ and for every natural number $k$ holds $F(k)$ is non-negative and $n \leq m$, then $\left(\sum_{\alpha=0}^{\kappa} F(\alpha)\right)_{\kappa \in \mathbb{N}}(n)(x) \leq$ $\left(\sum_{\alpha=0}^{\kappa} F(\alpha)\right)_{\kappa \in \mathbb{N}}(m)(x)$.

(38) Suppose $F$ has the same dom and $x \in \operatorname{dom} F(0)$ and for every natural number $m$ holds $F(m)$ is non-negative. Then $\left(\sum_{\alpha=0}^{\kappa} F(\alpha)\right)_{\kappa \in \mathbb{N}} \# x$ is nondecreasing and $\left(\sum_{\alpha=0}^{\kappa} F(\alpha)\right)_{\kappa \in \mathbb{N}} \# x$ is convergent.

(39) If for every natural number $m$ holds $F(m)$ is without $-\infty$, then $\left(\sum_{\alpha=0}^{\kappa} F(\alpha)\right)_{\kappa \in \mathbb{N}}(n)$ is without $-\infty$.

(40) If for every natural number $m$ holds $F(m)$ is without $+\infty$, then $\left(\sum_{\alpha=0}^{\kappa} F(\alpha)\right)_{\kappa \in \mathbb{N}}(n)$ is without $+\infty$.

(41) Suppose that for every natural number $n$ holds $F(n)$ is measurable on $E$ and $F(n)$ is without $-\infty$. Then $\left(\sum_{\alpha=0}^{\kappa} F(\alpha)\right)_{\kappa \in \mathbb{N}}(m)$ is measurable on E.

(42) Suppose that

(i) $F$ is additive and has the same dom,

(ii) $G$ is additive and has the same dom,

(iii) $\quad x \in \operatorname{dom} F(0) \cap \operatorname{dom} G(0)$, and

(iv) for every natural number $k$ and for every element $y$ of $X$ such that $y \in \operatorname{dom} F(0) \cap \operatorname{dom} G(0)$ holds $F(k)(y) \leq G(k)(y)$.

Then $\left(\sum_{\alpha=0}^{\kappa} F(\alpha)\right)_{\kappa \in \mathbb{N}}(n)(x) \leq\left(\sum_{\alpha=0}^{\kappa} G(\alpha)\right)_{\kappa \in \mathbb{N}}(n)(x)$.

(43) Let $X$ be a non empty set and $F$ be a sequence of partial functions from $X$ into $\overline{\mathbb{R}}$. If $F$ is additive and has the same dom, then $\left(\sum_{\alpha=0}^{\kappa} F(\alpha)\right)_{\kappa \in \mathbb{N}}$ has the same dom.

(44) Suppose that

(i) $\operatorname{dom} F(0)=E$,

(ii) $F$ is additive and has the same dom,

(iii) for every natural number $n$ holds $\left(\sum_{\alpha=0}^{\kappa} F(\alpha)\right)_{\kappa \in \mathbb{N}}(n)$ is measurable on $E$, and

(iv) for every element $x$ of $X$ such that $x \in E$ holds $F \# x$ is summable. 
Then $\lim \left(\left(\sum_{\alpha=0}^{\kappa} F(\alpha)\right)_{\kappa \in \mathbb{N}}\right)$ is measurable on $E$.

(45) Suppose that for every natural number $n$ holds $F(n)$ is integrable on $M$. Let $m$ be a natural number. Then $\left(\sum_{\alpha=0}^{\kappa} F(\alpha)\right)_{\kappa \in \mathbb{N}}(m)$ is integrable on $M$.

(46) Suppose that

(i) $E=\operatorname{dom} F(0)$,

(ii) $F$ is additive and has the same dom, and

(iii) for every natural number $n$ holds $F(n)$ is measurable on $E$ and $F(n)$ is non-negative and $I(n)=\int F(n) \mathrm{d} M$.

Then $\int\left(\sum_{\alpha=0}^{\kappa} F(\alpha)\right)_{\kappa \in \mathbb{N}}(m) \mathrm{d} M=\left(\sum_{\alpha=0}^{\kappa} I(\alpha)\right)_{\kappa \in \mathbb{N}}(m)$.

\section{Sequence of Measurable Functions}

Next we state two propositions:

(47) Suppose that

(i) $E \subseteq \operatorname{dom} f$,

(ii) $f$ is non-negative,

(iii) $f$ is measurable on $E$,

(iv) $F$ is additive,

(v) for every $n$ holds $F(n)$ is simple function in $S$ and $F(n)$ is non-negative and $E \subseteq \operatorname{dom} F(n)$, and

(vi) for every $x$ such that $x \in E$ holds $F \# x$ is summable and $f(x)=$ $\sum F \# x$.

Then there exists a sequence $I$ of extended reals such that for every $n$ holds $I(n)=\int F(n)\left\lceil E \mathrm{~d} M\right.$ and $I$ is summable and $\int f\left\lceil E \mathrm{~d} M=\sum I\right.$.

(48) Suppose $E \subseteq \operatorname{dom} f$ and $f$ is non-negative and $f$ is measurable on $E$. Then there exists a sequence $g$ of partial functions from $X$ into $\overline{\mathbb{R}}$ such that

(i) $g$ is additive,

(ii) for every natural number $n$ holds $g(n)$ is simple function in $S$ and $g(n)$ is non-negative and $g(n)$ is measurable on $E$,

(iii) for every element $x$ of $X$ such that $x \in E$ holds $g \# x$ is summable and $f(x)=\sum g \# x$, and

(iv) there exists a sequence $I$ of extended reals such that for every natural number $n$ holds $I(n)=\int g(n)\left\lceil E \mathrm{~d} M\right.$ and $I$ is summable and $\int f\lceil E \mathrm{~d} M=$ $\sum I$.

Let $X$ be a non empty set. Observe that there exists a sequence of partial functions from $X$ into $\overline{\mathbb{R}}$ which is additive and has the same dom.

Let $C, D, X$ be non empty sets, let $F$ be a function from $C \times D$ into $X \rightarrow \overline{\mathbb{R}}$, let $c$ be an element of $C$, and let $d$ be an element of $D$. Then $F(c, d)$ is a partial function from $X$ to $\overline{\mathbb{R}}$. 
Let $C, D, X$ be non empty sets, let $F$ be a function from $C \times D$ into $X$, and let $c$ be an element of $C$. The functor $\operatorname{curry}(F, c)$ yields a function from $D$ into $X$ and is defined as follows:

(Def. 6) For every element $d$ of $D$ holds $(\operatorname{curry}(F, c))(d)=F(c, d)$.

Let $C, D, X$ be non empty sets, let $F$ be a function from $C \times D$ into $X$, and let $d$ be an element of $D$. The functor $\operatorname{curry}^{\prime}(F, d)$ yields a function from $C$ into $X$ and is defined as follows:

(Def. 7) For every element $c$ of $C$ holds $\left(\operatorname{curry}^{\prime}(F, d)\right)(c)=F(c, d)$.

Let $X, Y$ be sets, let $F$ be a function from $\mathbb{N} \times \mathbb{N}$ into $X \dot{\rightarrow}$, and let $n$ be a natural number. The functor $\operatorname{curry}(F, n)$ yielding a sequence of partial functions from $X$ into $Y$ is defined by:

(Def. 8) For every natural number $m$ holds $(\operatorname{curry}(F, n))(m)=F(n, m)$.

The functor $\operatorname{curry}^{\prime}(F, n)$ yields a sequence of partial functions from $X$ into $Y$ and is defined by:

(Def. 9) For every natural number $m$ holds $\left(\operatorname{curry}^{\prime}(F, n)\right)(m)=F(m, n)$.

Let $X$ be a non empty set, let $F$ be a function from $\mathbb{N}$ into $(X \dot{\mathbb{R}})^{\mathbb{N}}$, and let $n$ be a natural number. Then $F(n)$ is a sequence of partial functions from $X$ into $\overline{\mathbb{R}}$.

The following four propositions are true:

(49) Suppose $E=\operatorname{dom} F(0)$ and $F$ has the same dom and for every natural number $n$ holds $F(n)$ is non-negative and $F(n)$ is measurable on $E$. Then there exists a function $F_{1}$ from $\mathbb{N}$ into $(X \dot{\rightarrow} \overline{\mathbb{R}})^{\mathbb{N}}$ such that for every natural number $n$ holds

(i) for every natural number $m$ holds $F_{1}(n)(m)$ is simple function in $S$ and $\operatorname{dom} F_{1}(n)(m)=\operatorname{dom} F(n)$,

(ii) for every natural number $m$ holds $F_{1}(n)(m)$ is non-negative,

(iii) for all natural numbers $j, k$ such that $j \leq k$ and for every element $x$ of $X$ such that $x \in \operatorname{dom} F(n)$ holds $F_{1}(n)(j)(x) \leq F_{1}(n)(k)(x)$, and

(iv) for every element $x$ of $X$ such that $x \in \operatorname{dom} F(n)$ holds $F_{1}(n) \# x$ is convergent and $\lim \left(F_{1}(n) \# x\right)=F(n)(x)$.

(50) Suppose that

(i) $E=\operatorname{dom} F(0)$,

(ii) $F$ is additive and has the same dom, and

(iii) for every natural number $n$ holds $F(n)$ is measurable on $E$ and $F(n)$ is non-negative.

Then there exists a sequence $I$ of extended reals such that for every natural number $n$ holds

$I(n)=\int F(n) \mathrm{d} M$ and $\int\left(\sum_{\alpha=0}^{\kappa} F(\alpha)\right)_{\kappa \in \mathbb{N}}(n) \mathrm{d} M=\left(\sum_{\alpha=0}^{\kappa} I(\alpha)\right)_{\kappa \in \mathbb{N}}(n)$.

(51) Suppose that

(i) $E \subseteq \operatorname{dom} F(0)$, 
(ii) $F$ is additive and has the same dom,

(iii) for every natural number $n$ holds $F(n)$ is non-negative and $F(n)$ is measurable on $E$, and

(iv) for every element $x$ of $X$ such that $x \in E$ holds $F \# x$ is summable.

Then there exists a sequence $I$ of extended reals such that for every natural number $n$ holds $I(n)=\int F(n)\lceil E \mathrm{~d} M$ and $I$ is summable and $\int \lim \left(\left(\sum_{\alpha=0}^{\kappa} F(\alpha)\right)_{\kappa \in \mathbb{N}}\right)\left\lceil E \mathrm{~d} M=\sum I\right.$.

(52) Suppose that

(i) $E=\operatorname{dom} F(0)$,

(ii) $F(0)$ is non-negative,

(iii) $F$ has the same dom,

(iv) for every natural number $n$ holds $F(n)$ is measurable on $E$,

(v) for all natural numbers $n, m$ such that $n \leq m$ and for every element $x$ of $X$ such that $x \in E$ holds $F(n)(x) \leq F(m)(x)$, and

(vi) for every element $x$ of $X$ such that $x \in E$ holds $F \# x$ is convergent.

Then there exists a sequence $I$ of extended reals such that for every natural number $n$ holds $I(n)=\int F(n) \mathrm{d} M$ and $I$ is convergent and $\int \lim F \mathrm{~d} M=$ $\lim I$.

\section{REFERENCES}

[1] Grzegorz Bancerek. The fundamental properties of natural numbers. Formalized Mathematics, 1(1):41-46, 1990.

[2] Grzegorz Bancerek. The ordinal numbers. Formalized Mathematics, 1(1):91-96, 1990

[3] Józef Białas. Infimum and supremum of the set of real numbers. Measure theory. Formalized Mathematics, 2(1):163-171, 1991.

[4] Józef Białas. Series of positive real numbers. Measure theory. Formalized Mathematics, 2(1):173-183, 1991.

[5] Józef Białas. The $\sigma$-additive measure theory. Formalized Mathematics, 2(2):263-270, 1991.

[6] Czesław Byliński. Binary operations. Formalized Mathematics, 1(1):175-180, 1990.

[7] Czesław Byliński. Functions and their basic properties. Formalized Mathematics, 1(1):5565, 1990.

[8] Czesław Byliński. Functions from a set to a set. Formalized Mathematics, 1(1):153-164, 1990.

[9] Czesław Byliński. Partial functions. Formalized Mathematics, 1(2):357-367, 1990.

[10] Czesław Byliński. Some basic properties of sets. Formalized Mathematics, 1(1):47-53, 1990.

[11] Noboru Endou and Yasunari Shidama. Integral of measurable function. Formalized Mathematics, 14(2):53-70, 2006.

[12] Noboru Endou, Yasunari Shidama, and Keiko Narita. Egoroff's theorem. Formalized Mathematics, 16(1):57-63, 2008.

[13] Noboru Endou, Katsumi Wasaki, and Yasunari Shidama. Basic properties of extended real numbers. Formalized Mathematics, 9(3):491-494, 2001.

[14] Noboru Endou, Katsumi Wasaki, and Yasunari Shidama. Definitions and basic properties of measurable functions. Formalized Mathematics, 9(3):495-500, 2001.

[15] Noboru Endou, Katsumi Wasaki, and Yasunari Shidama. The measurability of extended real valued functions. Formalized Mathematics, 9(3):525-529, 2001.

[16] P. R. Halmos. Measure Theory. Springer-Verlag, 1987.

[17] Andrzej Nȩdzusiak. $\sigma$-fields and probability. Formalized Mathematics, 1(2):401-407, 1990.

[18] Beata Padlewska. Families of sets. Formalized Mathematics, 1(1):147-152, 1990. 
[19] Beata Perkowska. Functional sequence from a domain to a domain. Formalized Mathematics, 3(1):17-21, 1992.

[20] Zinaida Trybulec. Properties of subsets. Formalized Mathematics, 1(1):67-71, 1990.

[21] Edmund Woronowicz. Relations defined on sets. Formalized Mathematics, 1(1):181-186, 1990.

[22] Hiroshi Yamazaki, Noboru Endou, Yasunari Shidama, and Hiroyuki Okazaki. Inferior limit, superior limit and convergence of sequences of extended real numbers. Formalized Mathematics, 15(4):231-236, 2007.

Received March 18, 2008 